La

Révolution

française

\section{La Révolution française}

Cahiers de l'Institut d'histoire de la Révolution française

5 | 2013

Le républicanisme anglais dans la France des

Lumières et de la Révolution

David V. Erdman, Commerce des Lumières. John

Oswald and the British in Paris, 1790-1793

Columbia, University of Missouri Press, 1986, 338 pages

Mathieu Ferradou

(2) OpenEdition

Journals

Édition électronique

URL : http://journals.openedition.org//rf/979

DOI : $10.4000 /$ Irf.979

ISSN : $2105-2557$

Éditeur

IHMC - Institut d'histoire moderne et contemporaine (UMR 8066)

Référence électronique

Mathieu Ferradou, "David V. Erdman, Commerce des Lumières. John Oswald and the British in Paris,

1790-1793 », La Révolution française [En ligne], 5 | 2013, mis en ligne le 31 décembre 2013, consulté le 23 septembre 2020. URL : http://journals.openedition.org//rf/979; DOI : https://doi.org/10.4000/lrf.

979

Ce document a été généré automatiquement le 23 septembre 2020.

(c) La Révolution française 


\title{
David V. Erdman, Commerce des Lumières. John Oswald and the British in Paris, 1790-1793
}

Columbia, University of Missouri Press, 1986, 338 pages

\author{
Mathieu Ferradou
}

\section{RÉFÉRENCE}

David V. Erdman, Commerce des Lumières. John Oswald and the British in Paris, 1790-1793, Columbia, University of Missouri Press, 1986, 338 pages.

1 " Oswald, vous avez vécu si longtemps sans goûter de chair que vous avez à présent un appétit des plus voraces pour le sang. » C'est ainsi que Thomas Paine aurait apostrophé son interlocuteur et ami lors d'une fête donnée par des membres de la Convention (probablement fin 1792 ou début 1793) alors qu'Oswald défendait l'idée que, face au risque de la guerre civile en France, il fallait mettre à mort tous les suspects. Cette anecdote, d'une véracité douteuse, est rapportée par Henry Redhead Yorke, ancien jacobin et révolutionnaire, qui avait côtoyé Oswald au sein du « club britannique » dont ils étaient tous deux membres, avant de revenir en Angleterre où, emprisonné pour conspiration entre 1795 et 1797, il retourna sa veste et dénonça ses anciennes relations ${ }^{1}$.

2 Que cette anecdote soit véridique ou non, à travers cette réprimande de Paine sont posées les questions-clés de l'ouvrage de David Erdman : le personnage de John Oswald, sa place dans la dynamique révolutionnaire des deux côtés de la Manche dans les années 1790-1793 et la question du radicalisme ${ }^{2}$. Ce radicalisme est ici à la fois politique et culturel : l'exécution des traitres dans la République menacée et la participation d'Oswald à l'entreprise constitutionnelle de 1792-1793 sont ainsi directement reliées à la question du végétarisme d'Oswald, auteur en 1791 de The Cry of Nature; or an Appeal to Mercy and to Justice, on behalf of the Persecuted Animals. Il s'agit pour Yorke de 
décrédibiliser la pensée politique de ce «fou » d'Oswald en montrant sa déviance tout comme les détracteurs d'Helena Maria Williams (qu'Oswald avait côtoyée) démontraient le danger de sa pensée en dénonçant sa sexualité prétendument débridée.

3 John Oswald - poète passionnément démocrate, soldat, journaliste politique et satiriste, pornographe à l'occasion', auteur d'Une Constitution pour la République universelle - est né vers 1760 à Edinburgh. En 1781, il embarqua pour l'Inde s'étant engagé dans l'armée anglaise. Son voyage fut l'occasion d'une halte de plusieurs mois dans l'île de Joanna dans l'archipel des Comores où il vécut parmi les habitants et où il eut une révélation : Mayotte refusait de payer le tribut à l'île de Joanna, affirmant "Mayotte comme Amérique $^{5}$." Arrivé en Inde, il combattit les troupes du sultan Hyder Ali allié aux Français et adopta le végétarisme qu'il associa au pythagorisme. Quittant l'armée et vendant sa commission, il effectua le voyage de retour par la voie de terre et séjourna parmi les Turcs et les Kurdes. De retour à Londres, il se mêla aux milieux populaires et radicaux du journalisme londonien et parisien, avant de séjourner de manière définitive à Paris à partir du printemps 1792. Il y rejoignit, nous démontre Erdman, les "Quatorze » planificateurs de la prise de la Bastille, puis participa aux activités du Cercle social et collabora au journal de Nicolas de Bonneville, La Bouche de fer avant de créer le Patriote universel (le pendant anglais du Patriote français de Brissot, publié en anglais, à Paris, afin d'échapper à la répression contre les écrits "séditieux " en Angleterre) qui parut à partir de 1790 mais qui fut rapidement supprimé et dont aucun exemplaire n'a survécu ${ }^{6}$. Il fut le premier étranger membre du club des Jacobins où il fut admis grâce à la publication, à Paris, de ses Remarques sur la Constitution de la GrandeBretagne en mai $1790^{7}$. En 1792, il fut également l'organisateur, avec l'approbation de Danton ${ }^{8}$, du premier régiment de piquiers volontaires (hommes et femmes à égalité) qui fut probablement aux premières loges de l'exécution de Louis XVI avant de partir pour la Vendée à l'été 1793 où Oswald fut tué dans un accrochage mineur. Oswald suscita de nombreuses polémiques de son vivant : il était végétarien, révolutionnaire, démocrate, républicain et « féministe ». Il fut même l'objet d'une légende après sa mort lorsque son éditeur londonien, William Thomson, qui avait publié ses travaux dans les années 1780, était persuadé que ce jeune général victorieux nommé Bonaparte qui émergeait sur le devant de la scène révolutionnaire en décembre 1793 ne pouvait être qu'Oswald sous une nouvelle identité. Pourtant, John Oswald, malgré ce parcours extraordinaire, est aujourd'hui largement méconnu voire totalement inconnu.

David V. Erdman (1911-2001), professeur de littérature, spécialiste de William Blake, a ainsi proposé, dans son livre publié en 1986 et injustement ignoré en France ${ }^{9}$, de sortir cette figure clé du radicalisme anglais et français de l'oubli historique et historiographique dans lequel il était plongé. John Goldworth Alger fut le premier à proposer, à la fin du XIX ${ }^{\mathrm{e}}$ siècle, un travail d'ampleur sur les Britanniques à Paris pendant la Révolution française. Ce travail, cependant, fortement marqué par l'historiographie britannique et sa vision très négative de la Révolution ${ }^{10}$, voyait en Oswald et les autres Britanniques des «traitres » à leur pays voire des criminels, des marginaux, seule explication possible pour comprendre leur enthousiasme pour la Révolution, synonyme de chaos et de bain de sang, surtout après les massacres de Septembre ${ }^{11}$. S'appuyant principalement sur des archives de journaux, mais aussi sur les sources imprimées contemporaines, Erdman esquisse une biographie quasifictionnelle mais toujours rigoureusement documentée de John Oswald dans laquelle il cherche à comprendre véritablement ce personnage, à lui redonner une dignité 
d'acteur de l'histoire, dans toute sa complexité face aux tumultes des années 1790-1793. Il tente également de saisir ce qui fait la cohérence de l'action et de la pensée d'Oswald : ce double mouvement du cosmopolitisme et du républicanisme. D'ailleurs - et c'est là l'un des principaux mérites de cet ouvrage - Erdman parvient à montrer que la question du végétarisme d'Oswald, que ses détracteurs soulignaient souvent, est à relier avec son cosmopolitisme et son républicanisme, les deux s'exprimant à travers le projet d'une république universelle.

5 Après une introduction dans laquelle Erdman annonce son intention de sortir Oswald de l'oubli dans lequel il a été maintenu, l'auteur consacre trois chapitres aux années de formation (de l'Ecosse à la côte de Malabar puis son voyage de retour et enfin ses activités journalistiques en Angleterre) puis un chapitre sur les liens d'Oswald avec Paris et, enfin, une série de chapitres sur ses activités radicales au sein des Jacobins ou au sein du club britannique qu'il contribue à organiser. L'originalité de son approche tient donc davantage dans les quatre «inter-chapitres» qui sont autant de chronologies servant à montrer les différents contextes - national et international sans lesquels les actions et la pensée d'Oswald ne peuvent être comprises. Ces chapitres donnent à la fois une dynamique à la lecture mais également une mise en perspective tout à fait stimulante qui éclaire efficacement le personnage.

6 La part quasi-fictionnelle vient de ce qu'Erdman n'hésite pas à faire vivre au lecteur, par un style très littéraire, les tribulations de son héros (et c'est bien ce qu'est Oswald sous la plume d'Erdman). Dans le même temps, Erdman ne cache pas les zones d'ombre, présente clairement les supputations nées du silence des sources, propose des hypothèses, s'interroge sous nos yeux, en avant-garde des questions qui se posent aujourd'hui sur les rapports entre histoire et fiction. Erdman suit la trace fugace d'Oswald, malgré les impasses, malgré le manque d'archives. Mieux: ce manque d'archives lui permet d'être passionnant car il nous montre par là même « l'atelier de l'historien ». Ainsi le lecteur conduit l'enquête en même temps que lui, lorsqu'il nous livre, bruts, les indices qu'il parvient à glaner dans les pages du London Gazetteer, du Herald ou celles de la Chronique du mois (autant de journaux auxquels Oswald a collaboré), dans les retranscriptions des séances du club des Jacobins, dans l'adresse à la Convention du club britannique de novembre 1792 ou dans les registres de marche du régiment lors de la campagne d'Oswald en Vendée dans laquelle il trouve la mort.

7 Erdman reconstitue ainsi l'univers mental, intellectuel et éditorial dans lequel Oswald évoluait. Ce livre est donc une véritable plongée dans les milieux cosmopolites et radicaux des patriotes réformateurs et révolutionnaires des années 1780 et 1790, qu'ils soient britanniques, français, hollandais ou même vénézuélien (avec le général Miranda). Outre Bonneville et Brissot déjà cités, on croise David Williams, Thomas Paine, Condorcet, Collot d'Herbois (dont Oswald traduit en anglais L'Almanach du Père Gérard, commentaire sur la Constitution de 1791), le "Citoyen Lord" Fitzgerald, organisateur de la Grande Rébellion irlandaise de 1798, Madgett, le traducteur irlandais au ministère des Affaires étrangères, Théroigne de Méricourt et bien d'autres dans des «cercles sociaux» fascinants qu'Oswald fréquentait et qu'il influençait, bien que n'étant que rarement au premier plan sauf lorsqu'il agit en tant que secrétaire du « club britannique » formé en 1792-1793.

8 Il en ressort un portrait presque en creux de ce John Oswald, polyglotte ${ }^{12}$, apôtre du végétarisme, soldat, citoyen français ${ }^{13}$, républicain théoricien et praticien d'une République universelle, qui voulait l'union des deux nations, française et anglaise, et 
qui se définissait comme "Anglo-Franc». Sa mort en Vendée lui a sans doute, selon Erdman, permit d'échapper aux désillusions qui ont suivi l'exaltation de la proclamation de la République et les projets « utopiques » qui ont fleuri avant d'être abandonnés face à la guerre entre la France et l'Angleterre puis face «au gouvernement révolutionnaire jusqu'à la paix » de décembre 1793. Ainsi, il n'a connu que de loin les mesures contre les Anglais d'août 1793, et n'a pas vécu pour assister à l'exclusion des femmes du club des Jacobins puis de la Convention. Il n'a pas vécu non plus pour connaître la Terreur, mais peut-être l'eût-il approuvée... ou en eût-il été une victime, étant donné son inimitié avec Robespierre.

9 Si parfois la qualité du style l'emporte sur la clarté des informations, si quelques erreurs sont perceptibles ${ }^{14}$, et si Erdman survalorise probablement le rôle d'Oswald au sein du « club britannique », ce livre est un tel plaisir de lecture, le produit d'une telle intelligence et d'une telle érudition de la littérature et des sources de l'époque, qu'il est un véritable modèle de production historique d'autant qu'il propose une écriture de l'histoire tout à fait stimulante. La double approche - historique et littéraire - qui soustend l'ouvrage est nettement perceptible. D'ailleurs, Erdman montre à quel point le personnage d'Oswald a pu influencer la littérature romantique à commencer par Wordsworth qui en fait un personnage de deux de ses pièces. Dans sa conclusion, Erdman écrit qu'il espère avoir redonné envie de s'intéresser à cet homme extraordinaire que fut Oswald et, plus largement, il explique qu'il s'agit pour lui de raviver l'attention des historiens sur le rôle des révolutionnaires qui ont formé le « club britannique ${ }^{15} »$.

10 Commerce des Lumières porte ainsi très bien son titre. Cette expression fut utilisée pour la première fois par Léonard Bourdon aux Jacobins, le 18 novembre 1791, pour qualifier la visite de Pétion à Londres au moment de la campagne électorale pour la commune de Paris : celle-ci fut donc entreprise "pour y faire commerce des lumières ${ }^{16}$.» Puisant dans les travaux de Robert Darnton sur la "bohème littéraire» ainsi que sur le mesmérisme, dans ceux de James Billington sur la «foi révolutionnaire » et de Gary Kates sur le Cercle social et les Girondins, Erdman replace Oswald dans cet « underground » littéraire, mêlé aux loges maçonniques des années 1780 à Londres, où il rencontra Brissot (dont il se méfia) et Bonneville (avec lequel il sympathisa) par l'intermédiaire du philosophe et prêtre gallois David Williams. Ce dernier avait d'ailleurs développé l'image des cercles constitutionnels, chaque constitution de l'histoire de la Grande-Bretagne permettant une plus ou moins grande liberté du peuple $^{17}$. Ce « commerce» retrace ainsi les cercles de plus en plus grands et complexes que les révolutionnaires formaient, l'image du cercle étant donc déterminante dans l'esprit de ces radicaux, leur idéal étant que ce cercle soit celui d'une République universelle, passant de la " société d'harmonie mesmérique à une harmonie sociale ${ }^{18}$.

11 Avec l'éclatement de la Révolution en France, ces intellectuels et hommes d'action britanniques, français, américains ou irlandais - ont tenté de penser le nouveau régime qui émergeait lorsque la Manche était une "voie de circulation dans les deux sens" pour ces "citoyens du monde ». Ils articulaient les idées de nation et de république universelle, de cosmopolitisme et d'égalité entre les hommes, les femmes et les anciens esclaves. Erdman montre comment le républicanisme d'Oswald doit être compris comme le cœur de sa pensée : son cosmopolitisme, son végétarisme, son "féminisme ", son militarisme également sont autant d'aspects de cette volonté de créer une république universelle, synonyme de « régénération » pythagoricienne et rousseauiste 
des individus et du corps social dans son ensemble ${ }^{19}$. La République, pour Oswald, est une république en marche et en armes (de piques, l'arme du peuple, qu'il préfère donc aux fusils), ce qui permet de faire des républicains, de révolutionner le peuple et de le mobiliser dans une entreprise véritablement démocratique et égalitaire. Erdman invite ainsi à une relecture de l'idéologie des Girondins et du Cercle social qui fut bien plus radicale que ne laisse penser l'étiquette de "modérés » qui leur est souvent apposée. Ces projets étaient d'autant plus radicaux qu'ils semblent à priori idéalistes et donc utopiques. Mais Erdman rappelle que dans l'esprit de ces révolutionnaires, écrire et penser cette révolution c'était la faire. Annoncer ces projets dans les pages de leurs journaux était une manière de faire advenir ces projets de régénération. Telle était la raison d'être de la Chronique $d u$ mois: faire en sorte que les idées deviennent évènements ${ }^{20}$.

Oswald avait théorisé ces rapports entre république, commerce et guerre dès les années 1786 lorsqu'il dénonçait, dans les colonnes du Herald et du British Mercury, le traité de commerce signé entre la France et l'Angleterre. Rappelant, dans un article intitulé "State of the World» du British Mercury" ${ }^{21}$, que les deux «moyens pour les nations de s'adresser et de correspondre les unes aux autres sont la guerre et le commerce ${ }^{22}$ ", Oswald fustigeait ce traité signé avec la France tyrannique tout en prédisant qu'il entrainerait la chute de l'Angleterre ainsi qu'une révolution en Grande-Bretagne voire en France dans le contexte d'une guerre européenne. Dans cette guerre, la Grande Bretagne devait épouser le parti de la liberté et s'allier avec les républiques hollandaise et américaine. On retrouve ici le modèle de la révolution et de la guerre d'indépendance américaines qui a tant influencé les radicaux dans les années 1780-1790: ce type de guerre est l'inverse des guerres menées jusqu'alors par les rois européens de concert avec les intérêts mercantiles qui ne servaient, «en Europe comme en Afrique » qu'à «faire des esclaves ${ }^{23}$."

13 Tout au contraire, le «commerce " prôné par Oswald, est un «commerce des lumières ", car il est à la fois mercantile, intellectuel et guerrier. Il est le moyen de régénérer l'ensemble de l'Europe (voire du monde) pour établir la république démocratique universelle. On peut ainsi mieux comprendre la manière dont Henry Redhead Yorke critiqua le projet oswaldien de constitution d'une démocratie universelle en lui reprochant d'avoir oublié les animaux :

Pleinement convaincu de ce principe, il [Oswald] propose de remodeler les gouvernements de toutes les nations du monde; les hommes et les femmes s'assembleront dans une plaine à ciel ouvert, et y feront, ou abrogeront leurs propres lois. J'ai souvent tenté de le convaincre que son plan n'était pas assez extensif, puisqu'il excluait de cette grande assemblée du monde animé la portion la plus importante de ses semblables, à savoir les chats, les chiens, les chevaux, les poules, \& $\mathrm{c}^{24}$.

14 Oswald, dans son essai sur le Gouvernement du Peuple; ou une Proposition pour une Constitution pour la République Universelle (imprimé en anglais à Paris fin 1792 ou début 1793) définissait en effet une constitution comme le moyen qui doit permettre à la volonté du peuple de s'exercer, réfutant ainsi l'idée que le système représentatif puisse être à même de remplir cet objectif. Hommes et femmes, sans distinction aucune, devaient être le fondement même de la république démocratique :

Nous ne pouvons pas exercer pour les autres les plus basses fonctions de l'existence animale ; et pouvons-nous alors accomplir pour les autres les plus hautes fonctions de la vie intellectuelle? Mais le fait est que, même si nous ne pouvons pas penser les uns pour les autres, pas plus que nous ne pouvons aimer les uns pour les autres, ou 
manger ou boire les uns pour les autres, pourtant, par habitude de déléguer à d'autres la tâche de penser pour nous, nous désapprenons insensiblement à penser ensemble, et cela répond merveilleusement à l'objectif charitable de ces Gentlemen qui sont prompts à nous épargner la peine de penser pour nous-mêmes ${ }^{25}$.

C'est cet essai qu'Edmund Burke, alarmé devant une telle audace, dénonce à la Chambre des Communes en mars 1793, prenant peur face à la remise en question par Oswald du système représentatif ainsi que de la propriété privée ${ }^{26}$, et ce, dans le contexte de la guerre entre la France et l'Angleterre depuis le $1^{\text {er }}$ février $1793^{27}$. Dès lors, comprendre la guerre que se livrent la France révolutionnaire et l'Angleterre monarchique, et notamment cette séquence - courte, mais cruciale - qui court de la chute de la monarchie en août 1792 jusqu'à la déclaration de guerre de février 1793 ne peut se faire sans prendre en compte ces radicaux britanniques et leurs liens avec les Girondins, ces « réseaux pluriels » au sein desquels Oswald jouait un rôle prédominant. Cette guerre est une guerre à la fois pour la prépondérance économique mais elle est aussi une guerre idéologique, l'affrontement de deux modèles qui paraissent alors antithétiques. C'est dans cette optique qu'Erdman propose une thèse intéressante mais peut-être encore fragile : les radicaux britanniques et irlandais - Paine, Oswald et Fitzgerald en tête - auraient cherché à provoquer cette guerre entre l'Angleterre et la France à l'automne 1792 puis à l'hiver 1792-1793 afin de forcer la main à cette dernière qui n'aurait eu d'autre choix que d'envoyer une force militaire en Angleterre et/ou en Irlande, ce qui aurait permis à ces deux pays d'être "révolutionnés » (puisque l'armée française y aurait reçu un soutien populaire ${ }^{28}$ ), établissant ainsi une république anglaise et une république irlandaise ${ }^{29}$. Cette tentative expliquerait la rupture entre Oswald et les Brissotins, au début de 1793, ces derniers n'ayant pas voulu cette guerre.

Le mérite du livre de David Erdman est de rappeler et de permettre de comprendre, en fin de compte, pourquoi Oswald et, avec lui, l'ensemble de ces radicaux qui circulaient entre les deux rives de la Manche ont soit été oubliés soit condamnés à la damnatio memoriae : la victoire historique de l'Angleterre dans les French Wars fut également une victoire historiographique qui n'est remise en question que depuis quelques dizaines d'années. A ce titre, il est regrettable que ce beau livre n'ait pas été traduit.

\section{NOTES}

1. Henry Redhead YORKE, Letters from France in 1802, London, 1804, vol. I, p. 162, cité par David V. ERDMAN, Commerce des Lumières, p. 8 (les traductions proposées ici sont les nôtres).

2. Le "radicalisme" désignant, en anglais, à partir de 1797, les partisans (dont Charles James Fox) d'une « réforme radicale » de la franchise électorale au Parlement britannique mais qui fut portée dès le début de la Révolution française par les sociétés comme la London Corresponding Society fondée en mars 1792 par Thomas Hardy et dont la première règle était « That the number of our Members be unlimited », promesse de démocratie républicaine éminemment subversive dans l'Angleterre aristocratique de George III. Les termes de " radicalisme » et de "radicaux » se sont imposés à partir du début du $\mathrm{XIX}^{\mathrm{e}}$ siècle. Auparavant, ces radicaux sont désignés comme «patriotes» ou « jacobins ». L'ouvrage fondateur en ce domaine reste Edward Palmer THOMPSON, 
La Formation de la classe ouvrière anglaise, Paris, Le Seuil, 2012 (édition de poche, première traduction française, 1988, première édition anglaise 1963).

3. Publié, à Londres, avec cette indication: « by John Oswald, member of the Club des Jacobines ».

4. Oswald dénonça la folie de George III et sa proclamation contre les imprimeurs d'écrits ou d'images choquantes (qui rappelle les mesures répressives des années 1792-1794) sur le ton de la farce, multipliant les allusions scabreuses et priapiques, le tout illustré par les caricatures (non signées) de James Gillray (ERDMAN, op. cit. p. 52-56 et 65-71).

5. Erdman cite ici l'un des onze essais intitulés «The Present Times " publiés dans The Herald entre 1785 et 1788, et attribués, en partie, à Oswald (p. 48-49).

6. ERDMAN, op. cit. p. 109-116 sur la signification quais-mystique de ces « Quatorze » organisateurs de la Commune de Paris puis quatorze éditeurs de la Chronique du mois.

7. Composant une ode en hommage à la Révolution, "Le Triomphe de la Liberté ", qu'il dédie à l'Assemblée nationale lors de son ouverture le 4 septembre 1790, Oswald cite avec fierté son appartenance à la Société des Amis de la Constitution (ERDMAN, op. cit. p. 81).

8. ERDMAN, op. cit. p. 134.

9. Cette notice est, à notre connaissance, la première dans une revue française.

10. Voir Pascal DupuY, «La diffusion des stéréotypes révolutionnaires dans la littérature et le cinéma anglo-saxons (1789-1989)», Annales historiques de la Révolution française, n³05, 1996, p. 511-528.

11. Voir, notamment: John Goldworth ALGER, Englishmen in the French Revolution, London, Ballantyne Press, 1889 et "The British Colony in Paris, 1792-1793," The English Historical Review, 1898, p. 672-694.

12. Lors de son voyage de retour de l'Inde, Oswald est devenu, selon ses propres dires, «familier avec le langage arabique, ainsi qu'avec les dialectes français, italien, espagnol et portugais » tandis que David Stewart, en 1825, prétendait que, durant le voyage de l'Angleterre à l'Inde, Oswald avait « acquis une connaissance du grec, de l'hébreu et des langues gaéliques » (ERDMAN, op. cit., p. 12 et 20 ).

13. Oswald reçut la citoyenneté française de la Convention, en même temps que Thomas Cooper, John Horne Tooke, Thomas Christie et Joseph Warner - autant de radicaux - le 25 septembre 1792, après que 18 étrangers dont sept Britanniques l'aient déjà reçus de l'Assemblée législative juste après la chute de la monarchie. Voir Mike RAPPORT, « "Deux nations malheureusement rivales" : les Français en Grande-Bretagne, les Britanniques en France, et la construction des identités nationales pendant la Révolution française », AHRF, n 342 , octobredécembre 2005, p. 21-46. Ainsi, Mike Rapport rappelle cette déclaration du dantoniste François Chabot à propos de Joseph Priestley : il était « cosmopolite, par conséquent français ».

14. Ainsi, Erdman localise l'hôtel White où a eu lieu le dîner inaugural du club britannique le 18 novembre 1792 rue des Piques (p. 218) alors qu'il était 7, passage des Petits Pères. De plus, Erdman lorsqu'il évoque Nicholas Madgett (p. 262) se trompe sur plusieurs points : le plaçant (à juste titre) au centre des manœuvres menées en vue d'une expédition française en Irlande au début de l'année 1793, il commet plusieurs erreurs en citant l'ouvrage de Lionel D. Woodward, Une adhérente anglaise de la Révolution française : Helen Maria Williams et ses amis, Paris, H. Champion, 1930, p. 102n. qui lui-même s'appuyait sur une lettre de Madgett à Lebrun ministre des Affaires étrangères (voir Archives diplomatiques, Correspondance Politique Angleterre $587 \mathrm{f}^{\circ} 45-46$ ) et se trompe sur l'âge de Madgett (en 1793, Madgett a environ 53 ans et non 26).

15. Si l'historiographie anglo-saxonne, depuis les années 1980, a effectivement renouvelé son intérêt pour les radicaux britanniques (voir notamment pour une mise au point le $n^{\circ} 342$ d'octobre-décembres des Annales historiques de la Révolution française consacré aux «Iles britanniques et la Révolution française »), toutefois, en France, seule Rachel ROGERS, dans sa thèse (en anglais) Vectors of Revolution: The British Radical Community in Early Republican Paris, 1792-1794 
(Université Toulouse-Le Mirail, 2012) a répondu à cet appel, explorant les radicaux britanniques et leur implication, notamment dans l'œuvre constitutionnelle de 1793 mais aussi dans leurs récits de la Révolution et donc leur contribution aux représentations de la Révolution en Angleterre.

16. ERDMAN, op. cit. p. 139 et 143. A noter que Bourdon, par la suite, lorsqu'il se trouve à Hambourg en 1797-1798 comme agent du Directoire, cherche lui aussi à organiser ce commerce des lumières notamment en encourageant la formation d'un comité révolutionnaire irlandais chargé de la propagande vers l'Irlande en vue d'un second débarquement, après l'échec de celui de 1796.

17. ERDMAN, op. cit. p. 76. Voir également Robert DARNTON, Bohème littéraire et Révolution, Paris, Gallimard, 2010 [1983 pour la première traduction française, 1982 pour la première édition en anglais] ; Robert DARNTON, La fin des Lumières. Le mesmérisme et la Révolution, Paris, Odile Jacob, 1995 [1970, 1968] ; James H. BILLINGTON, Fire in the Minds of Men : Origins of the Revolutionary Faith, New York, Basic Books, 1980 ; Gary KATES, The Cercle Social, the Girondins, and the French Revolution, Princeton, Princeton University Press, 1985 ; David Williams, Lessons to a Young Prince, by an Old Stateman, on the present disposition in Europe to a General Revolution, London, H. D. Symonds, 1790 (comportant quatre diagrammes représentant les différentes constitutions anglaises que Bonneville réimprima dans la Bouche de fer et utilisa dans son De l'esprit des religions en 1791).

18. ERDMAN, op. cit. p. 74-76.

19. ERDMAN, op. cit. p. 33.

20. ERDMAN, op. cit. p. 130.

21. Oswald fut le principal auteur et l'éditeur du Mercury pour les deux mois de son éphémère existence en mai-juin 1787, financé par James Ridgway (peut-être avec le soutien de l'opposition whig), celui qui publie l'Address to the Addressers de Thomas Paine en 1792 (en réponse aux attaques contre son Rights of Man) dans lequel il appelle de ses vœux une révolution en GrandeBretagne (ERDMAN, op. cit. p. 42 et 78).

22. ERDMAN, op. cit. p. 79.

23. ERDMAN, op. cit. p. 98.

24. ERDMAN, op. cit. p. 7

25. ERDMAN, op. cit., p. 293.

26. Dès 1790 , dans le discours inaugural qu'il prononce le 20 novembre à la Society for Free Debate à Londres, en défense à la pétition du radical John Horne Tooke, Oswald dénonce la corruption du Parlement, celui-ci n'était pas représentatif puisque ses membres sont soit une aristocratie (et donc «monstrueux » par nature) soit élus par une part infime du peuple, les propriétaires. Il dénonce ainsi le principe selon lequel la propriété est une condition pour accéder au vote. Pour Erdman, Oswald s'inspire ici de son compatriote écossais, William Ogilvie, auteur d'un Essay on the Right of Property in Land, publié à Londres en 1781 (ERDMAN, op. cit. p. 91-101). En cela, Oswald a parfois été considéré, à la suite de Ogilvie, et avant Claude Fauchet Bonneville, comme un précurseur du socialisme (ERDMAN, op. cit. p. 116-117).

27. ERDMAN, op. cit., p. 272.

28. Or, dans cette perspective, l'Angleterre et l'Irlande connaissaient une situation bien différente: alors que le loyalisme conservateur, orchestré par le pouvoir, était populaire en Angleterre, le radicalisme et l'espoir d'une révolution l'emportaient en Irlande. Voir AHRF, $\mathrm{n}^{\circ} 342$ déjà cité ainsi que Jim SMYTH, "Introduction: the 1798 rebellion in its eighteenth-century contexts", dans Jim SMYTH (dir.), Revolution, Counter-revolution and Union: Ireland in the 1790s, Cambridge, Cambridge University Press, 2000, p. 1-20, notamment p.12-13 où Smyth rappelle qu'en Irlande, « les journaux radicaux se vendaient plus que les journaux pro-gouvernementaux, les Defenders et les Irlandais Unis étaient plus nombreux que les Orangistes, et les Droits de l'Homme de Paine circulaient encore plus largement qu'en Grande-Bretagne. La réception plus favorable des classes inférieures irlandaises au message radical suggère une cohésion sociale et 
une stabilité politique moindres en Irlande qu'en Grande-Bretagne ainsi qu'une semblable faiblesse de l'argumentaire conservateur et de l'hégémonie élitaire. » Sur l'immense popularité de Paine en Irlande, voir David DICKSON, «Paine and Ireland », dans David DICKSON, Dáire KEOGH, Kevin WHELAN (dir.), The United Irishmen. Republicanism, Radicalism and Rebellion, Dublin, Lilliput Press, 1993, p. 135-150.

29. Erdman développe cette hypothèse dans les chapitres 7 (où il examine l'action d'Oswald, son éloquence, aux Jacobins où il s'oppose notamment à Robespierre) et 8 (sur le « club britannique), p. 187-243. 